\title{
School dinners, are they meeting the standards?
}

\author{
L. McGuffin ${ }^{1}$, T. A. McCrorie ${ }^{1}$, J. McBratney ${ }^{2}$ and H. McCarthy ${ }^{1}$ \\ ${ }^{1}$ NICHE, University of Ulster, Coleraine BT52 1SA, UK and ${ }^{2}$ Public Health Agency, Belfast BT2 8HS, UK
}

School lunches contribute significantly to a child's daily intake, with around one-third being provided by one meal ${ }^{(1)}$. In 2008 , the School Food Trust (SFT) began to implement 'Food-and Nutrient-Based' standards (FNS) in primary schools in England, however, Northern Ireland (NI) currently follow 'Food-Based' standards. NI must now evaluate whether changing to FNS is required to improve school meals.

Three primary schools in NI provided 3-week menu cycles, recipes and number of portion sizes sold for nutrient analysis (WISP, v3.0 Tinuviel Software Ltd, UK). Statistical analysis was carried out using SPSS (v15, SPSS Inc, USA) to compare the nutritional content of NI primary school meals with the SFT's 'Nutrient-Based' standards (NBS) ${ }^{(2)}$.

\begin{tabular}{|c|c|c|c|c|c|c|c|c|c|}
\hline \multirow[b]{2}{*}{ Energy (kcal) } & \multicolumn{2}{|c|}{$\frac{\text { All Schools }}{(n 3)}$} & \multicolumn{2}{|c|}{$\frac{\text { School A }}{(n 60)}$} & \multicolumn{2}{|c|}{$\frac{\text { School B }}{(n 40)}$} & \multicolumn{2}{|c|}{$\frac{\text { School C }}{(n \text { 185) }}$} & \multirow{2}{*}{$\begin{array}{c}\begin{array}{c}\text { Nutrient-based } \\
\text { standard }^{(2)}\end{array} \\
530 \pm 26.5\end{array}$} \\
\hline & $520^{1}$ & $(425,613)$ & $463^{a}$ & $(394,563)$ & 507 & $(427,638)$ & 572 & $(461,697)$ & \\
\hline Protein $(g)$ & $23.3^{\mathrm{b}}$ & $(19.9,29.4)$ & $20.6^{\mathrm{b}}$ & $(16.5,28.5)$ & $23.3^{\mathrm{b}}$ & $(20.8,29.0)$ & $25.9^{\mathrm{b}}$ & $(21.1,30.1)$ & Min 7.5 \\
\hline Fat (g) & $17.8^{b}$ & $(12.9,25.7)$ & $16.6^{b}$ & $(11.9,23.1)$ & $19.9^{b}$ & $(12.9,25.7)$ & $16.2^{\mathrm{b}}$ & $(13.0,30.4)$ & Max 20.6 \\
\hline Saturated fat (g) & 5.7 & $(4.6,7.6)$ & 5.2 & $(3.8,7.5)$ & 5.7 & $(4.1,7.5)$ & 6.2 & $(4.7,9.4)$ & Max 6.5 \\
\hline Carbohydrate (g) & 65.6 & $(53.1-82.1)$ & $54.9^{\mathrm{a}}$ & $(47.8,74.2)$ & 61.8 & $(57.0,80.9)$ & 78.8 & $(63.0,102.9)$ & Min 70.6 \\
\hline NME (g) & $6.7^{\mathrm{b}}$ & $(2.7,12.3)$ & $8.3^{\mathrm{c}}$ & $(6.4,14.0)$ & $6.4^{\mathrm{c}}$ & $(0.5,14.4)$ & $4.8^{\mathrm{b}}$ & $(2.3,7.5)$ & Max 15.5 \\
\hline Calcium (mg) & 198 & $(145,254)$ & 151 & $(121,201)$ & 199 & $(171,263)$ & 214 & $(168,300)$ & Min 193 \\
\hline Sodium (mg) & $573^{\mathrm{c}}$ & $(389,827)$ & 521 & $(286,755)$ & 518 & $(377,728)$ & $684^{\mathrm{c}}$ & $(21,30)$ & Max 499 \\
\hline Iron $(\mathrm{mg})$ & $2.6^{\mathrm{c}}$ & $(2.2,3.1)$ & $2.3^{\mathrm{c}}$ & $(1.9,3.0)$ & $2.4^{\mathrm{c}}$ & $(2.2,2.9)$ & 3.0 & $(2.7,3.7)$ & Min 3.0 \\
\hline Folate (mg) & 55 & $(40,65)$ & 41 & $(37,61)$ & 57 & $(46,68)$ & 61 & $(45,72)$ & Min 53 \\
\hline Vitamin C (mg) & $20.2^{\mathrm{b}}$ & $(13.1,26.4)$ & $14.4^{\mathrm{c}}$ & $(11.3,22.6)$ & $21.6^{\mathrm{b}}$ & $(13.9,26.2)$ & $23.9^{\mathrm{b}}$ & $(18.2,29.2)$ & Min 10.5 \\
\hline Fibre $(\mathrm{g})$ & $4.8^{\mathrm{c}}$ & $(3.5,6.3)$ & 4.0 & $(3.3,5.6)$ & 4.9 & $(3.6,5.7)$ & $5.9^{\mathrm{c}}$ & $(3.8,6.6)$ & Min 4.2 \\
\hline
\end{tabular}

${ }^{\mathrm{abc}}$ Median values with a superscript are significantly different from the 'Nutrient Based' standard using the Wilcoxon-signed rank test: ${ }^{\mathrm{a}} P<0.05,{ }^{\mathrm{b}} P<0.001,{ }^{\mathrm{c}} P<0.01$.

NME, Non-milk extrinsic sugars.

Overall, there were no significant differences between the NBS and the school menus, with the exception of Fe being significantly lower $(P<0.01)$ and Na being significantly higher $(P<0.01)$. School C significantly exceeded the Na NBS $(P<0.01)$, although it was the only school that met the recommendations for Fe.

These analyses illustrate that primary schools in NI are likely to be providing meals which meet the SFT's NBS, while following the current 'Food-Based' standards. Hence, a change of standards may not be required. However, further evaluation of the school menus in a larger sample of primary schools is required along with a review of the standard recipes specifically for $\mathrm{Na}$ and $\mathrm{Fe}$ content. In addition, an investigation of the actual food consumed by school children will provide more accurate information to determine whether school children are meeting their requirements through school meals.

1. Gregory J, Lowe S, Bates C et al. (2000) National Diet and Nutrition Survey-Young People Aged 4-18 Years Old, vol. 1. London: The Stationery Office.

2. School Food Trust (2008a) Nutrient Based Standards. http://www.schoolfoodtrust.org.uk/content.asp?ContentId=641 (Accessed 2 October 2008). 